\title{
Inactivation du gène NF2 dans les tumeurs sporadiques
}

I a neurofibromatose du type 2 (NF2), dont le signe le plus fréquent est un neurinome de l'acoustique, est une maladie autosomique dominante. Son gène, localisé en $22 q 12$, a été identifié en 1993 par deux équipes et appelé merline [1] ou schwannomine [2] $\left(\mathrm{m} / \mathrm{s} n^{\circ} 4\right.$, vol. 9, p. 484). Ce gène s'étend sur au moins $50 \mathrm{~kb}$; la protéine compte 587 acides aminés; deux messagers principaux donnent des bandes de 2,6 et $7 \mathrm{~kb}$.

La pathologie de la NF2 suggérait pour la protéine un rôle de suppresseur de tumeurs que les deux mêmes équipes se sont efforcées de préciser; mais, en outre, elles ont étendu leurs investigations à des cas de tumeurs non familiaux; leurs résultats paraissent dans un même numéro de Nature Genetics [3, 4]. I.e principe de départ de ces travaux est que la perte d'un ou des deux allèles de la protéine pourrait s'observer en dehors des schwannomes vestibulaires qui sont le support habituel de la maladie. En particulier, la moitié de ces malades présentent un ou plusieurs méningiomes, spinaux ou crâniens. Ruttledge et al. [3] (Montréal, Canada, Stockholm et Gothenburg, Suède, et Paris, France), ont analysé 151 méningiomes sporadiques, parmi lesquels $60 \%$ avaient subi la perte d'un allèle, et étaient mononosomiques pour le chromosome 22. Une analyse de la séquence a été pratiquée sur les transcrits de ces méningiomes, portant sur 8 des 16 exons que compte le gène. I.es méthodes incluaient la SSCP (single strand conformation polymorphism), suivie éventuellement de séquençage. Dans vingt-quatre cas, des mutations furent observées; toutes les mutations étaient inactivatrices (anomalies d'épissage, décalages de phase ou codons de terminaison). I.es mutations se limitaient à la tumeur, l'ADN général restait normal. Bien entendu, le nombre réel des mutations est plus élevé pour deux raisons: la SSCP ne détecte pas toutes les mutations, mais environ $75 \%$; par ailleurs, la moitié des exons n'a pas été criblée, pas plus que les régions flanquantes.

Au total, les auteurs évaluent à $40 \%$ de l'ensemble les méningiomes dans lesquels NF2 n'est pas impliqué. D'autres anomalies chromosomiques ont en effet été décrites dans des cultures provenant de méningiomes et d'autres gènes responsables restent à découvrir.

Bianchi et al. [4] (Princeton, NJ, Charlestown, MA, Philadelphie, PA, Cincinnati, OH USA) ne se sont pas bornés à l'étude de schwannomes et de méningiomes, dans lesquels ils ont confirmé l'existence de mutations somatiques. Forts de la constatation, déjà faite antérieurement [1], que les transcrits du gène de la merline étaient retrouvés dans la plupart des tissus, même en dehors du système nerveux, ils ont analysé des tumeurs qui paraissaient sans rapport direct avec NF2; ils y ont été conduits par l'observation de la perte de chromosomes 22 dans plusieurs néoplasmes. Deux exemples sont particulièrement démonstratifs: (1) des mélanomes dérivent de la crête neurale embryonnaire, mais ne sont pas fréquents chez les malades atteints de NF2. Sur 3 mélanomes primaires et 17 métastases, des mutations du transcrit NF2 ont été trouvées six fois. En revanche, la recherche est restée vaine dans 5 cas de phéochromocytome; (2) sur 14 carcinomes du sein, une délétion provoquant une terminaison précoce a été observée une fois. Mais sur 20 cas de cancer colique, aucune anomalie n'a pu être détectée.

Les résultats des deux groupes montrent que l'altération du produit du gène NF2 est loin d'être confinée aux cas héréditaires de neurofibromatose du type 2. Elle est d'abord répandue dans les formes sporadiques atteignant les tissus frappés classiquement par la NF2, schwannomes et méningiomes. Débordant ces limites, elle peut frapper des tissus hors du système nerveux, avant tout les mélanomes, et parfois - rarement - les cancers du sein. Encore fautil envisager que certaines mutations ne soient pas causales, mais un effet tardif des remaniements cancéreux. Un caractère fondamental est que les anomalies n'atteignent que le tissu cancéreux et non l'ADN général. Dans la tumeur, selon le schéma classique des suppresseurs de tumeur, deux étapes sont nécessaires : la perte d'hétérozygotie par disparition d'un allèle, suivie de l'inactivation par mutation de l'allèle restant. Il s'agit, en effet, presque toujours de délétions ou de codons nonsens aboutissant à des protéines tronquées. A cet égard, Bianchi et al. [4] insistent sur l'importance probable de la région C-terminale ; ils ont mis en évidence une isoforme nouvelle, probablement due à un épissage alternatif, qui diffère de l'isoforme principale par les 16 derniers acides aminés, et qui existe aussi chez la souris ; ces isoformes pourraient être pathologiques. Il reste que le problème que nous posions l'an dernier $\left(\mathrm{m} / \mathrm{s} n^{\circ}\right.$, vol. 9, p. 484) reste entier: la merline-schwannomine ressemble à des protéines de membrane et d'adhérence connues; or on ne sait toujours pas en quoi un tel rôle explique la fonction de suppresseur de tumeur que semble exercer cette protéine en physiopathologie.

J.C.D.

1. Trofatter JA, Mac Collin R, Rutter JL, et al. A novel moesin-ezrin-radixin-like gene is a candidate for the neurofibromatosis 2 tumor suppressor. Cell 1993; 72: 791-800.

2. Rouleau (;A, Mérel P, Lutchman M, et al. Alteration of a new gene encoding a putative membrane-organizing protein causes neurofibromatosis type 2. Nature 1993; 363 : 515-21. 3. Ruttledge $M$ H, Sarrazin J, Rangaratnam S, et al. Evidence for the complete inactivation of the NF2 gene in the majority of sporadic meningiomas. Nature Genet 1994; 6: 180-4. 4. Bianchi $\mathrm{AB}$, Hara $\mathrm{T}$, Ramesh V, et al. Mutations in transcript isoforms of the neurofibromatosis 2 gene in multiple human tumour types. Nature Cienet 1994; $6: 185-92$. en cause dans certaines formes
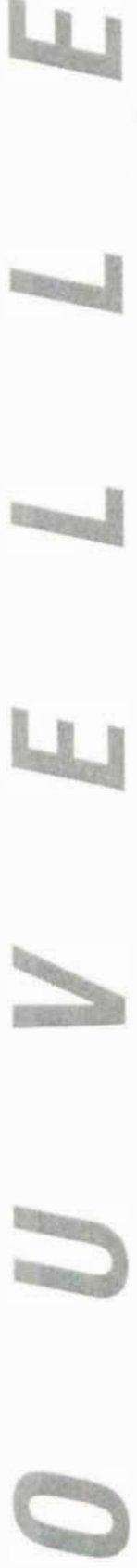

$\mathrm{m} / \mathrm{s} n^{\circ}$ 6-7 vol. 10, juin-juillet 94

. 